\title{
Effects of First Trimester Binge Alcohol Exposure on Developing White Matter in Fetal Sheep
}

\author{
HIROFUMI WATARI, DONALD E. BORN, AND CHRISTINE A. GLEASON \\ Department of Pathology-Neuropathology [H.W., D.E.B.], Department of Pediatrics [C.A.G.], University of Washington, \\ Seattle, Washington 98195
}

\begin{abstract}
Heavy prenatal alcohol exposure is associated with neurodevelopmental abnormalities. Neuropathologic and neuroimaging studies have shown a wide range of structural problems, including abnormal neuronal migration and volume reduction in specific brain regions, including white matter. We identified foci of significant fetal white matter microglia-macrophage immunoreactivity in a "binge" model of early prenatal alcohol exposure in sheep. Ewes of alcohol-exposed fetuses received daily $90 \mathrm{~min}$ alcohol $(1.5 \mathrm{gm} / \mathrm{kg}$ i.v.) infusions at $30-60 \mathrm{~d}$ gestation (term $=147 \mathrm{~d}$ ). Ewes of control fetuses received same volume infusions of normal saline intravenously. Near-term (125 d gestation) fetal brains were labeled with microglia-macrophages using HAM56 antibody. We quantified dense immunoreactive cellular regions across sections and anatomical locations using computer-assisted microscopy and quantitative morphometry. The proportional HAM56-positive area in cortical white matter was greater in the alcohol-exposed fetuses $(1.6 \%)$ compared with the saline controls $(0.7 \%)$. The areas were localized to the frontal gyral white matter, temporal gyral white matter, optic radiation, and others (corpus callosum, septum pellucidum, fasciculus subcallosus, and external capsule), with a greater distribution in the gyral white matter. The greater area of macrophage-rich regions in near-term fetal sheep brain suggests a vulnerability of developing white matter that is enhanced by early alcohol exposure. (Pediatr Res 59: 560-564, 2006)
\end{abstract}

$\mathrm{F}_{\mathrm{r}}$ AS is one of the leading yet preventable causes of mental retardation in the United States, and alcohol-related neurodevelopmental abnormalities contribute further to the morbidity associated with heavy prenatal alcohol exposure $(1,2)$. Clinical neuropathologic and neuroimaging studies done on FAS patients at different ages have shown a wide spectrum of neuroanatomical abnormalities, including white matter lesions (3-7). These white matter abnormalities have been described in corpus callosum, cerebellar vermis, optic nerves, hippocampus, basal ganglia, and temporal, parietal, and occipital white matter. There have been limited animal studies of prenatal alcohol exposure focused on white matter lesions. We developed a binge model of early, moderate prenatal alcohol exposure in sheep and we noted increased cortical white matter lesions on routine hematoxylin and eosin histology in near-

Received April 12, 2005; accepted November 17, 2005

Correspondence: Christine A. Gleason, M.D., UW Pediatrics Box 356320, 1959 NE Pacific Street, Seattle, WA 98195-6320; e-mail: cgleason@u.washington.edu

Supported, in part, by the Arc of Washington Trust Fund (C.A.G.), National Institutes of Health grant 5R01AA12403 (C.A.G.), and the National Institute of Child Health and Human Development (Grant P30 HD02274). term fetuses, compared with saline controls. The sensitive, selective microglia-macrophage marker HAM56 (human alveolar macrophage) (8) is an excellent indicator of inflammation, prior necrosis, or augmented programmed cell death. We used this marker, combined with computer-assisted microscopy and quantitative morphometry, to examine the effects of moderate binge prenatal alcohol exposure on the developing fetal sheep brain, specifically, the white matter.

\section{METHODS}

Maternal infusion protocol. All animal care and experimental study procedures were approved by the University of Washington Institutional Animal Care and Use Committee. Pregnant ewes were brought to the animal care facility at approximately $25 \mathrm{~d}$ gestation and remained there until the fetuses were removed at $125 \mathrm{~d}$ gestation (term $=147 \mathrm{~d}$ ). The ewes were divided into two groups (alcohol and saline control). Ewes in each group received intravenous infusions via an external jugular vein catheter. Ewes in the alcohol group received $1.5 \mathrm{~g} / \mathrm{kg}$ of pure ethanol (AAPER Alcohol and Chemical Co., Shelbyville, KY) diluted 1:3 in normal saline (Baxter, Toronto, ON, Canada; infusion volume $=5.7 \mathrm{~mL} / \mathrm{kg}$ ) infused daily (Monday-Friday) over a 1.5-h interval. Ewes in the saline control group received an equivalent volume of saline $(5.7 \mathrm{~mL} / \mathrm{kg})$ during the same time interval. Infusions were performed $5 \mathrm{~d}$ per week for a period of $4 \mathrm{wk}$ beginning on approximately $\mathrm{d}$ 30 and ending on d 60 of gestation, for a total of $20 \mathrm{~d}$ of infusion. Blood alcohol levels were obtained twice weekly immediately before and after alcohol infusions in both groups. The ewes were provided with feed and water ad libitum, including during all infusions.

Fetal tissue and immunohistological preparation. On approximately $\mathrm{d}$ 125 of gestation, the pregnant ewes were killed with an overdose of sodium pentobarbital (Abbott Laboratories, North Chicago, IL) and the fetuses were removed from the uterus via a hysterotomy. The carotid arteries were cannulated and the fetal brains were perfused with heparinized saline $(1: 1$; American Pharmaceutical Partners, Inc., Schaumburg, IL) and then $10 \%$ neutral buffered formalin (Fisher Scientific, Fair Lawn, NJ). The brains were removed, weighed, and immersed in formalin for 2-3 d. Brains were then cut into two hemispheres along the falx cerebri. The left hemisphere was then sectioned in the coronal plane into 105 -mm-thick blocks using a specially designed guide to ensure equal thickness of the sections. These tissue blocks were dehydrated through increasing concentrations of ethanol $(70 \%, 95 \%$, and 100\%), processed through xylene (JT Baker, Inc., Phillipsburg, NJ), and infiltrated with paraffin. The embedded tissues were sectioned at six microns thickness on a rotary microtome (Leica, Heidelberg $\mathrm{GmbH}$, Mannheim, Germany), taking the first section that contained the complete tissue in the tissue block, and mounted on glass slides (Fisher Scientific, Pittsburgh, PA). If the section was damaged later in processing, the next section was taken and mounted.

Abbreviations: FAS, fetal alcohol syndrome; PVL, periventricular leukomalacia

DOI: 10.1203/01.pdr.0000203102.01364.de 
Hematoxylin (Sigma Chemical Co.-Aldrich, Inc., St. Louis, MO) and eosin stain was applied to each section from each block as a reference. For immunohistochemistry, sections on slides were deparaffinized, rehydrated through graded alcohols, treated with $1 \%$ peroxide (Sigma Chemical Co.Aldrich), heated to boiling for $10 \mathrm{~min}$ in $0.1 \mathrm{M}$ sodium citrate (JT Baker, Inc., Phillipsburg, NJ), followed by conditioning solution of 3\% BSA (BoehringerMannheim, Indianapolis, IN; Roche Diagnostics Corporation, Indianapolis, IN) in $0.1 \mathrm{M}$ solution sodium phosphate (JT Baker, Inc.). Antibody HAM56 (DAKO Corp., Carpinteria, CA) was applied at 1:500 dilution at room temperature for $1 \mathrm{~h}$ followed by antibody detection using $\mathrm{ABC}$ kit (Vector Laboratories, Burlingame, CA) and diaminobenzidine (Sigma Chemical Co.Aldrich) as chromagen.

Data analysis and calculations. We outlined the contour of each section by tracing the pial and ventricular surfaces (Fig. 1). Only one fetal brain from any pregnancy was analyzed. The outline of areas containing numerous immunoreactive microglia-macrophages and the entire outline of each brain section was traced in a blinded fashion using computer-assisted microscopy and quantitative morphometry. Because individual cells were marked by the label, this technique was less subject to variations of intensity of immunoreactivity. Each outlined area of microglia-macrophages was labeled according to its anatomical location with the aid of sheep brain atlases as a reference $(9-11)$. We defined gyral white matter as regions superficial to the depths of sulci and the remaining white matter regions were designated as "deep" except for specific regions including optic radiation, corpus callosum, septum pellucidum, fasciculus subcallosus, and external capsule. We used conventional anatomic landmarks to distinguish the various lobes. Software (Stereo Investigator, version 4.10d; MicroBrightField Laboratories, LLC., Williston, VT; ) and hardware (Lucivid, MicroBrightField Laboratories, LLC.) were set up with a Leitz Diaplan microscope and $2.5 \times$ lens (Leitz, Wetzlar, Germany). The data management and statistical analysis was done using commercially available software (Statistica, version 5.1 and 6.1; Statsoft Inc., Tulsa, OK; Excel 97 and higher, Microsoft Corporation, Redmond, WA).

The proportional microglia-macrophage rich cellular area (\% lesion) was calculated in three different ways: for the whole brain, for each section, and for anatomical location. The proportional microglia-macrophage-rich cellular area for a brain was calculated as \% lesion = total areas of HAM56 staining / total brain contour areas $\times 100 \%$; the proportional microglia-macrophagerich cellular area by section was calculated as $\%$ lesion by section $=$ area of HAM56 staining/ area of brain section $\times 100 \%$; lastly, the proportional microglia-macrophage cellular area by anatomical location was calculated as $\%$ lesion by topography $=$ sum of anatomically relevant area of HAM56 staining / total brain contour areas $\times 100 \%$. Differences were considered significant if $p<0.05$. All measurements were expressed as mean \pm SEM.

The effect of group on the \% brain lesion or anatomical region analyses was evaluated using the $t$ test (Figs. 2, 4, and 5). The effect of group-bysection was evaluated using repeated measures ANOVA with two factors (Fig. 3). In the two-way interaction repeated measures ANOVA, group (alcohol and saline) was the between-animal factor and section (1 through 10; Fig. 3) was the within-animal factor. Each \% section lesion was adjusted using logarithmic transformation by adding $1 \%$ and taking the natural $\log$, i.e. $\ln (\%$ lesion $+1 \%)$. The logarithmic transformation was used to reduce the
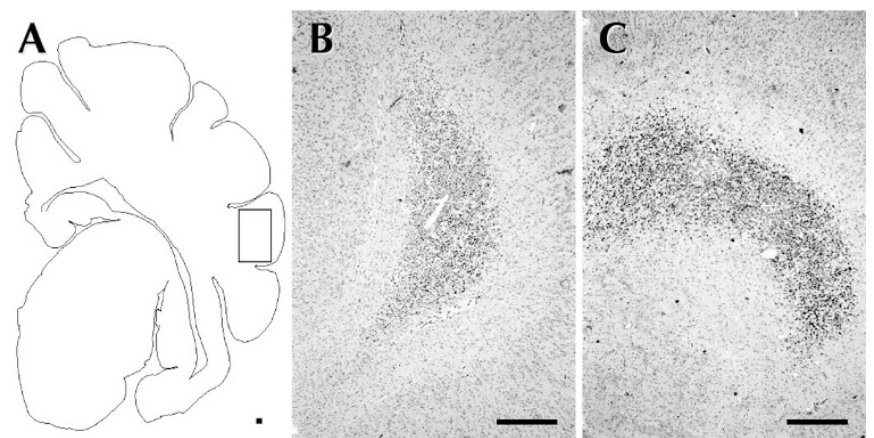

Figure 1. Representative sections of fetal sheep brain stained using HAM56 antibody that labels microglia-macrophages. (A) Image of computer-assisted contour trace of coronal fetal sheep brain section; left hemisphere, section 6 . (B) Photomicrograph of microglia-macrophage (HAM56 marker) in salineand $(C)$ alcohol-exposed fetuses, sampled from a square region designated in $A$. The dark area represents HAM56-positive cells. Both alcohol-exposed and saline control fetuses showed foci of numerous HAM56 immunoreactive cells identifying microglia-macrophage-rich regions, but the alcohol-exposed fetuses had proportionally more. The horizontal bar in each panel is $500 \mu \mathrm{m}$.

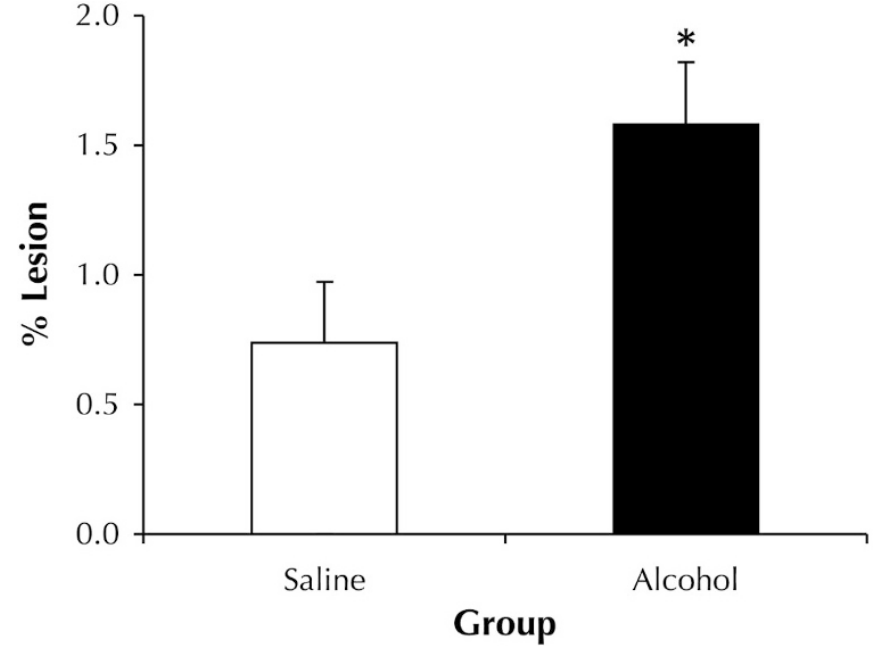

Figure 2. Comparison of proportional microglia-macrophage-rich cellular area (\% lesion) between groups. Alcohol-exposed fetuses $(n=7)$ show a greater lesion $(1.58 \pm 0.68 \%)$ than saline control $(n=8)(0.74 \pm 0.74 \%)$. (*p $<0.05)$. The bars represent SEM.

mean-variance relationship typical of proportional (percentage) data. After the transformation, the variance appeared approximately constant across all magnitudes of response, such that it met a key assumption of ANOVA. The addition of $1 \%$ to $\%$ lesion values fixed the "overcorrection" done by logarithmic transformation alone [i.e. $\ln (\%$ lesion)]. The validity of transformation was checked by the residual analysis. If the $F$ test was significant, the individual group differences were evaluated using a post hoc least significant difference (LSD) test.

\section{RESULTS}

Fifteen fetal brains ( 9 male, 6 female) obtained from 14 pregnant ewes were studied at $125 \pm 0.4 \mathrm{~d}$ gestation (mean \pm SEM) $($ term $=147 \mathrm{~d}$ gestation). The gestational age, body weight, brain weight, and brain-to-body weight ratio for each group are summarized in Table 1. There were no significant differences between the groups. In the alcohol group, average maternal blood alcohol concentration immediately after the infusion was $201 \pm 31 \mathrm{mg} / \mathrm{dL}$.

Representative sections of microglia-macrophage-rich cellular regions marked by HAM56 antibody in the left temporal gyral white matter are shown in Figure 1. Both alcohol and saline groups showed dense HAM56 staining (dark area in the light gray background) in the white matter (Fig. 1, $B$ and $C$ ). These cellular regions were commonly triangular to teardropshaped, approximately conforming to the shape of the subcortical/gyral white matter. Macrophage-rich regions in deeper white matter were more variable, sometimes rather linear half moon or star shaped, generally related to the shape of the particular white matter region involved. Qualitatively, there were more numerous and larger microglia-macrophage-rich cellular regions in the alcohol group. The packed appearance of the microglia-macrophages allowed a contour to be traced around the area of the region, and, thus, we were able to represent the quantity of region as an area (designated as \% lesion; see "Methods" for formulas). There were also individual macrophages sparsely distributed in white matter. Qualitatively, these were not different between the groups and any difference between groups in sparsely distributed macrophages was not further analyzed. 


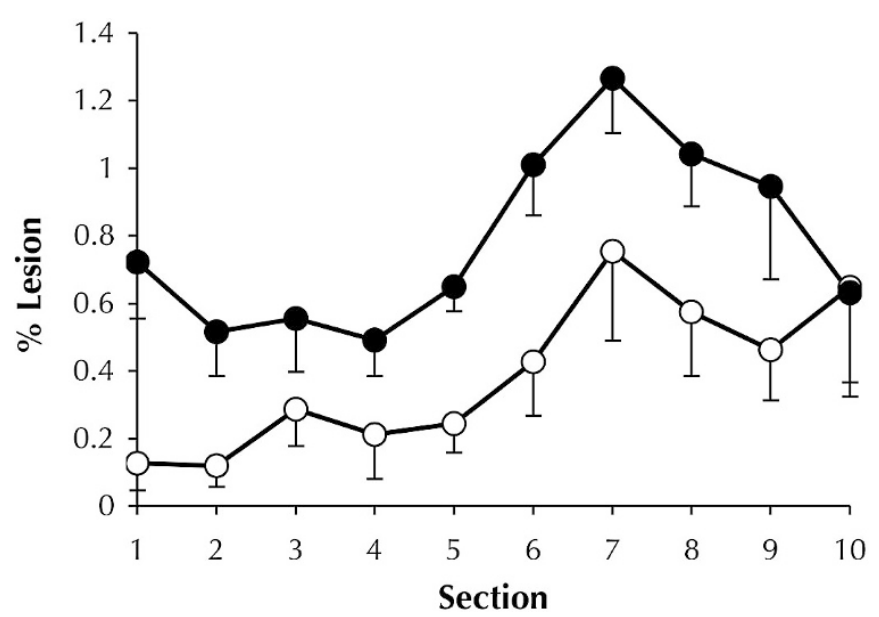

Figure 3. Distribution of proportional microglia-macrophage-rich cellular area (\% lesion) on the rostrocaudal axis of the brain. Alcohol-exposed ( $)$ fetuses $(n=7)$ showed greater \% lesion caudally (sections $5-10)$ and rostrally (sections 1-5; but to a lesser extent) than saline control $(\bigcirc)$ fetuses $(n=8)$. Each point on the figure (open circle, saline; filled circle, alcohol) represents the mean values of $\%$ lesion on a coronal section. Two-way interaction ANOVA with repeated measures showed $\mathrm{F}_{9,117}=0.81, p<0.61$. The bars represent SEM.

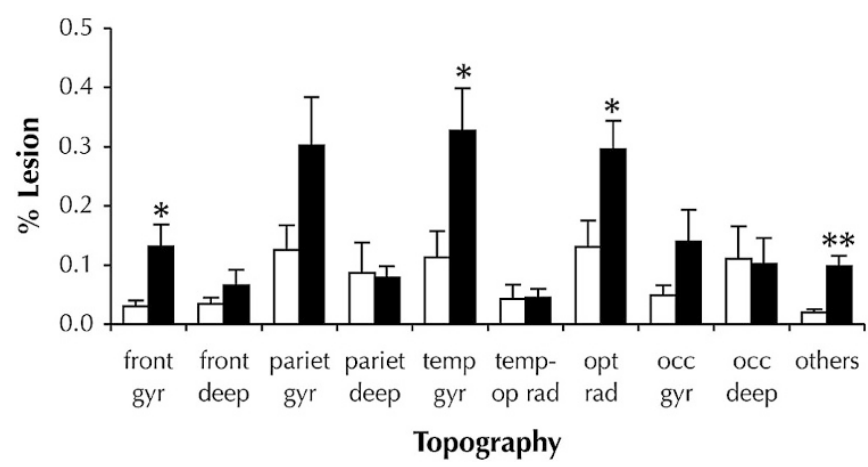

Figure 4. Comparison of the topography of proportional microgliamacrophage-rich cellular area (\% lesion) between groups. Each bar (open bar, saline; filled bar, alcohol) represents the mean values of \% lesion in a location. The bar on top represents + SEM. $* p<0.05$. $* * p<0.001$. Alcohol group ( $\square$ ) fetuses $(n=7)$, compared with saline group $(\square)$ fetuses $(n=8)$, show significantly greater $\%$ lesions in the frontal and temporal gyral white matters $(p<0.05, p<0.05)$, optic radiation $(p<0.05)$, as well as "others" $(p<0.001)$, which includes corpus callosum, fasciculus subcallosus, septum pellucidum, and external capsule.

To compare the amount of white matter microgliamacrophage-rich lesions between groups, we plotted $\%$ brain lesion versus group (Fig. 2). By group comparison $t$ test, there was a greater overall \% brain lesion area in the alcoholexposed fetuses compared with saline controls $(1.58 \pm 0.68 \%$ versus $0.74 \pm 0.74 \%)(p<0.05)$. Because alcohol-exposed fetal brains showed a greater proportional area of microgliamacrophage infiltration, we further analyzed the distribution of lesions across brain sections. We plotted \% brain lesion versus section on a rostrocaudal axis (from section 1 to 10) of the brain (Fig. 3). The figure shows that more microgliamacrophage rich regions are localized caudally (sections 6 through 10) than rostrally (sections 1 through 5) in both alcohol and saline groups. As expected from the previous analysis, the two-way interaction ANOVA with repeated mea-

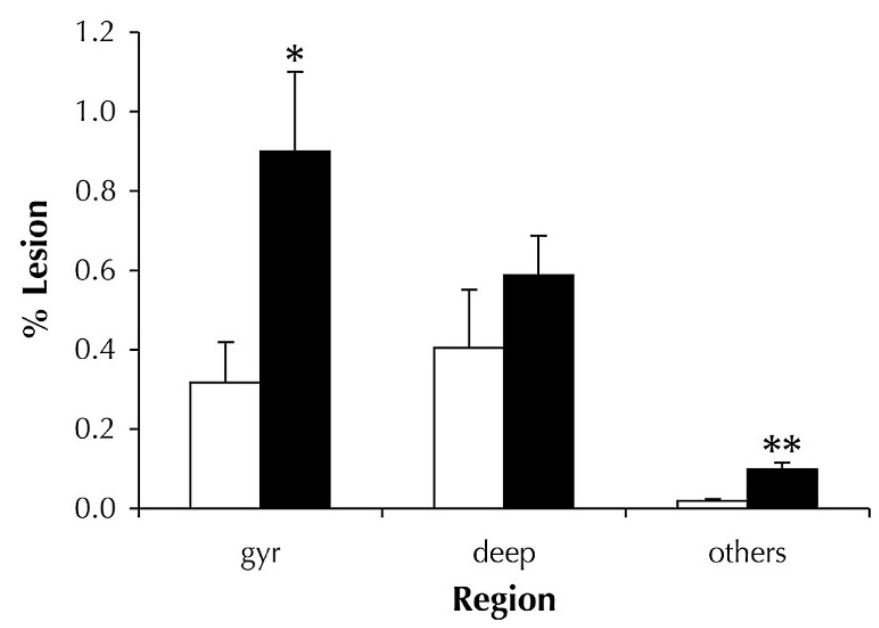

Figure 5. Comparison of proportional microglia-macrophage-rich cellular area (\% lesion) between the gyral white matter, deep white matter, and "others" between groups. Alcohol group ( $\square$ ) fetuses $(n=7)$ show significantly greater lesion in both the gyral white matter and others compared with saline group ( $\square$ ) fetuses ( $n=8 ; p<0.05$ and $p<0.001$, respectively). The bar on top represents + SEM. $* p<0.05 ; * * p<0.001$.

Table 1. Gestational age, body weight, brain weight, and brain weight-to-body weight ratio of fetal sheep exposed to daily intravenous alcohol binges in the first trimester $(n=7)$ or same volume saline controls $(n=8)$

\begin{tabular}{lcc}
\hline & $\begin{array}{c}\text { Alcohol } \\
(n=7)\end{array}$ & $\begin{array}{c}\text { Saline control } \\
(n=8)\end{array}$ \\
\hline Gender & 4 males & 3 males \\
Twins & 2 twins & 1 twin \\
Gestational age (d) & $125.6 \pm 0.6$ & $125.5 \pm 0.6$ \\
Body weight $(\mathrm{kg})$ & $2.53 \pm 0.26$ & $3.21 \pm 0.21$ \\
Brain weight $(\mathrm{g})$ & $43 \pm 1.6$ & $47.10 \pm 1.3$ \\
Brain weight:body weight & $0.018 \pm 0.004$ & $0.015 \pm 0.002$ \\
\hline
\end{tabular}

Only one animal from any pregnancy is included. The $t$ test shows $p>0.05$ between groups for body weight $(p=0.0599)$, brain weight $(p=0.0675)$, and brain-to-body weight ratio $(p=0.209)$.

sures (with the logarithmic transformation as described in "Methods") showed a significant difference in overall group comparison; $\mathrm{F}_{1,13}=6.37, p<0.05$. The novel finding in this analysis is that there is a significant difference in the section comparison; $\mathrm{F}_{9,117}=5.01, p<0.001$. The group-by-section interaction test showed no significant difference between the groups; $\mathrm{F}_{9,117}=0.81, p<0.61$.

We also defined anatomical distribution of microgliamacrophage-rich regions (from left to right: frontal gyral white matter, frontal deep white matter, parietal gyral white matter, parietal deep white matter, temporal gyral white matter, temporal-optic radiation, optic radiation, occipital gyral white matter, occipital deep white matter and "others," which include corpus callosum, septum pellucidum, fasciculus subcallosus, and external capsule) (Fig. 4). The anatomical analysis confirms that nearly all the microglia-macrophage-rich regions are in the white matter; an exception is the fasciculus subcallosus, which is a germinal matrix that represents a small proportion of overall microglia-macrophage-rich area. $t$ Tests showed significantly greater proportional microglia-macrophage area in the frontal gyral white matter $(p<0.05)$, temporal 
gyral white matter $(p<0.05)$, optic radiation $(p<0.05)$, and "others" $(p<0.001)$ in the alcohol-exposed group.

Our detailed anatomical analysis of microglia-macrophage distribution suggests that there is a greater proportional area of microglia-macrophage in the gyral white matter of alcoholexposed fetuses. To confirm this, we categorized microgliamacrophage-rich regions of the frontal, parietal, temporal, occipital lobes, and optic radiation into either gyral or deep white matter. The gyral white matter is found superficially, or in the gyrus, with respect to the deep white matter, which is found in the deeper regions of the hemisphere. Because microglia-macrophage rich cellular areas in "others" were not in the cortex, they were kept separate from the groupings. The result is plotted in Figure 5. As in the previous analysis, the "others" show a greater proportional microglia-macrophagerich region in the alcohol group fetuses $(p<0.001)$. More importantly, the alcohol group fetuses showed significantly greater proportional microglia-macrophage-rich region in the gyral white matter $(p<0.05)$ but not in the deep white matter.

\section{DISCUSSION}

The major finding of this study is that prenatal alcohol exposure during the first trimester in a pattern that mimics moderate binges produces microglia-macrophage-rich cellular regions in specific white matter foci of the fetal sheep brain. We used an immunohistochemical marker of macrophages as a sensitive way to identify these regions. Macrophage-rich regions were present focally in the white matter with a similar rostrocaudal distribution pattern in both alcohol and saline control fetuses, but the proportion of involved area was significantly greater in alcohol-exposed fetuses. Furthermore, a greater proportion of cellular regions were localized to specific anatomical locations of the alcohol-exposed brains, including frontal gyral white matter, temporal gyral white matter, optic radiation, and "others," including corpus callosum, septum pellucidum, fasciculus subcallosus, and external capsule. The proportional microglia-macrophage-rich area was biased in the alcohol group toward gyral white matter. The greater magnitude of microglia-macrophage-rich area in alcohol-exposed fetuses suggests that there is an effect of early alcohol exposure on developing white matter. Although we did not address the causal mechanisms, this effect could involve inflammation, necrosis, and/or augmentation of programmed cell death (apoptosis).

White matter damage and cellular reaction is a common form of neonatal brain injury and is the hallmark of periventricular leukomalacia (PVL) seen in preterm infants (12). Like PVL, alcohol exposure in the pattern of moderate binges produced multi-focal necrosis of the white matter (13). Our study revealed that one of the greatest areas of white matter lesions was in the optic radiation (Fig. 4). Infants with PVL who have retrogeniculate white matter visual pathway damage often have abnormal ophthalmologic outcomes, including subcortical visual loss, esotropia, and optic nerve hypoplasia (14). Clinically, the majority of FAS patients are known to exhibit ocular and visual anomalies, with up to $76 \%$ showing optic nerve hypoplasia (15). However, the general distribution of our lesions was not similar to the distribution of PVL lesions (16). And, unlike the topography of white matter damage in PVL, our alcohol-exposed fetuses had lesions distributed in both the gyral and deep white matter, with a significantly greater proportion in the former (Fig. 5).

Previous studies described alcohol-related lesions in various brain regions $(5,6,17,18)$. The topographical analysis in our study (Fig. 4) shows that prenatal alcohol exposure causes enhanced white matter macrophage-rich areas in specific regions of the brain. These regions are the frontal gyral white matter, temporal gyral white matter, optic radiation and "others," which includes corpus callosum, septum pellucidum, fasciculus subcallosus, and external capsule. The lesions in the temporal, parietal, and occipital white matter are consistent with the finding reported by Archibald et al. (5) of decreased white matter volume in these structures in the brains of children with a history of prenatal alcohol exposure. However, in contrast to previous studies showing alcohol-associated basal ganglia damage (5), we did not observe cellular regions highlighted by macrophage immunoreactivity in this location. It is possible that either neuronal damage in the basal ganglia does not produce a macrophage response or that prior macrophage responses had resolved by the time of our neuropathological study. A methodological consideration is that we used a simple marker for one cell type, which probably does not reflect the true extent of pathology. Various mechanisms of injury are still possible. It is also possible that there is not significant normal programmed cell death in these regions.

There are several complexities associated with the method of area measurements we used in our study: 1) difficulty identifying the border of a lesion; 2) missing tissue on a section; and 3) tissue expansion during slide preparation. First, we used a sensitive, selective immunohistochemical marker for microglia-macrophage (HAM56) (8) to identify inflammation, loss of myelin, necrosis, or accentuated apoptosis. However, the cellularity of microglia-macrophages can vary within a lesion or be so sparse so that it is difficult to see the boundary of the lesion. We selected only dense microglia-macrophagerich cellular regions that were visible at low power. The error introduced by this method is assumed to be small because the proportion of sparse regions is small compared with dense regions. Second, was the issue of missing tissue from a section. The edge of a brain tissue was missing on some slides due to histologic preparation. The consequence of this was that small sections toward the frontal and occipital poles may have an overestimate of microglia-macrophage-rich regions. When a large microglia-macrophage-rich region is present on a section and part of the nonimmunoreactive areas is missing, we may overestimate the \% lesion because the brain contour is small. A relatively large SEM in the alcohol and saline groups on section 10 may be explained by this effect (Fig. 3). And third, during preparation of sections from paraffin blocks, expansion during slide preparation could have introduced errors; the section is rehydrated and expands to a variable extent, changing the relative size of the sections. We used proportional data (i.e. \% lesion) as an appropriate solution to this problem, thus compensating for the changes in the overall section area due to tissue expansion. 
In conclusion, moderate binge prenatal alcohol exposure during the first trimester is associated with a greater magnitude of microglia-macrophage-rich white matter regions in specific locations of near-term fetal sheep brain. The magnitude and topography of the microglia-macrophages in both saline and alcohol-exposed fetuses suggests an inherent vulnerability of developing brain white matter, which is enhanced by exposure to alcohol. Although there are similarities to other models of white matter injury, the nature of the cellular response, the extent of vulnerable periods and pathogenesis of the observed white matter changes following exposure to alcohol in the developing fetal brain are yet to be elucidated.

Acknowledgments. The authors thank Dean Billheimer for his expert assistance on statistical analysis. Expert histologic services were provided by Randy Small and Christine Ulness, University of Washington Center for Human Development and Disability.

\section{REFERENCES}

1. Abel EL, Sokol RJ 1986 Fetal alcohol syndrome is now leading cause of mental retardation. Lancet 2:1222

2. Chan DQ 1999 Fetal alcohol syndrome. Optom Vis Sci 76:678-685

3. Roebuck TM, Mattson SN, Riley EP 1998 A review of the neuroanatomical findings in children with fetal alcohol syndrome or prenatal exposure to alcohol. Alcohol Clin Exp Res 22:339-344
4. Sowell ER, Thompson PM, Mattson SN, Tessner KD, Jernigan TL, Riley EP, Toga AW 2001 Voxel-based morphometric analyses of the brain in children and adolescents prenatally exposed to alcohol. Neuroreport 12:515-523

5. Archibald SL, Fennema-Notestine C, Gamst A, Riley EP, Mattson SN, Jernigan TL 2001 Brain dysmorphology in individuals with severe prenatal alcohol exposure. Dev Med Child Neurol 43:148-154

6. Clarren SK, Alvord Jr, EC Sumi SM, Streissguth AP, Smith DW 1978 Brain malformations related to prenatal exposure to ethanol. J Pediatr 92:64-67

7. National Institute on Alcohol Abuse and Alcoholism NIH 2000 Prenatal exposure to alcohol. Alcohol Res Health 24:32-41

8. Hulette CM, Downey BT, Burger PC 1992 Macrophage markers in diagnostic neuropathology. Am J Surg Pathol 16:493-499

9. Yoshikawa T 1968 Atlas of the Brains of Domestic Animals. University of Tokyo Press, Tokyo, pp S1-S23

10. Wilkie J 1937 The Dissection and Study of the Sheep Brain, as an Introduction to the Study of the Human Brain. Oxford University Press, London, pp 32-58, 84-92

11. Crosby EC, Schnitzlein HN 1982 Comparative Correlative Neuroanatomy of the Vertebrate Telencephalon. Macmillan Publishing Co., New York, pp 501-524

12. Holzman C, Paneth N, Little R, Pinto-Martin J 1995 Perinatal brain injury in premature infants born to mothers using alcohol in pregnancy. Neonatal Brain Hemorrhage Study Team. Pediatrics 95:66-73

13. Deguchi K, Oguchi K, Matsuura N, Armstrong DD, Takashima S 1999 Periventricular leukomalacia: relation to gestational age and axonal injury. Pediatr Neurol 20:370-374

14. Brodsky MC, Fray KJ, Glasier CM 2002 Perinatal cortical and subcortical visual loss: mechanisms of injury and associated ophthalmologic signs. Ophthalmology 109:85-94

15. Strömland K 1985 Ocular abnormalities in the fetal alcohol syndrome. Acta Ophthalmol 171:1-50

16. Volpe JJ 2001 Neurobiology of periventricular leukomalacia in the premature infant. Pediatr Res 50:553-562

17. Mallard EC, Williams CE, Johnston BM, Gluckman PD 1994 Increased vulnerability to neuronal damage after umbilical cord occlusion in fetal sheep with advancing gestation. Am J Obstet Gynecol 170:206-214

18. Karhunen PJ, Erkinjuntti T, Laippala P 1994 Moderate alcohol consumption and loss of cerebellar Purkinje cells. BMJ 308:1663-1667 